\title{
La diversité culturelle. une approche communicationnelle
}

Cultural Diversity: a communicational Approach

\section{Philippe Bouquillion}

\section{(2) OpenEdition}

12 Journals

Édition électronique

URL : http://journals.openedition.org/questionsdecommunication/1823

DOI : 10.4000/questionsdecommunication. 1823

ISSN : 2259-8901

Éditeur

Presses universitaires de Lorraine

\section{Édition imprimée}

Date de publication : 1 juillet 2008

Pagination : 251-268

ISBN : 978-2-86480-952-4

ISSN : 1633-5961

Référence électronique

Philippe Bouquillion, "La diversité culturelle. une approche communicationnelle », Questions de communication [En ligne], 13 | 2008, mis en ligne le 01 juillet 2010, consulté le 08 avril 2021. URL: http://journals.openedition.org/questionsdecommunication/1823; DOI : https://doi.org/10.4000/ questionsdecommunication.1823 


\section{$>$ NOTES DE RECHERCHE}

PHILIPPE BOUQUILLION

Centre d'études des médias, des technologies et de l'internationalisation

Université Paris 8 Vincennes-Saint-Denis

p.bouquillion@libertysurf.fr

\section{LA DIVERSITÉ CULTURELLE. UNE APPROCHE COMMUNICATIONNELLE}

Résumé. - La thématique de la diversité culturelle est au cœur de différentes
stratégies d'acteurs sociaux, liées à deux importantes mutations sociales: les
transformations du système interétatique et de l'État nation, dans un cadre de
mondialisation, ainsi que les articulations entre les industries des contenus et
les industries de la communication (télécommunications, Web et matériels). Les
théories et les discours de la diversité, œuvrant comme des « discours de vérité »
inséparables de systèmes de pouvoirs, accompagnent, facilitent et légitiment
quatre tendances de long terme qui marquent l'économie des contenus: le poids
croissant de l'aval des filières, l'organisation des industries de la culture et de la
communication dans une logique d'économie-monde, la polarisation des contenus
entre hits et contenus de niche, la remise en cause des politiques publiques.

Mots clés. - Diversité, industries de la culture, communication, Web, politiques culturelles, mondialisation. 
$\mathrm{P}$ ourquoi la thématique de la diversité culturelle suscite-t-elle aujourd'hui un tel intérêt de la part des pouvoirs publics nationaux, des instances supranationales et des chercheurs? L'histoire peut apporter des éléments de réponse. La question des flux culturels internationaux est très ancienne, comme l'ont rappelé Joëlle Farchy et Jean Tardif. Dès les années 20, les États européens et d'abord l'Allemagne, puis la Grande-Bretagne et plus tardivement la France (Mattelart, 2005 : 30) développent des dispositifs de protection de leur cinémal. Cette thématique est à nouveau d'actualité, après la Libération avec les accords Blum-Byrnes. Le troisième temps est celui qui précède les accords de Marrakech de 1994, fondant l'Organisation mondiale du commerce (OMC). Ces périodes présentent un double point commun : la place de certains États dans le système interétatique est remise en question, tandis que l'économie du cinéma connaît de profonds bouleversements. On observe la poursuite sous une forme accentuée de ce double mouvement depuis la mise en place de l'OMc, et surtout, depuis la déclaration universelle de 2002 et la convention de 2005, adoptées dans le cadre de l'UNESCO.

D'une part, le système interétatique est fortement bouleversé. Les institutions politiques ou financières, créées après la Seconde Guerre mondiale, semblent impuissantes à instaurer des nouvelles relations politiques et culturelles négociées, et à faire face aux partisans d'un choc des civilisations. L'OMC est décrite, à tort ou à raison, dans les discours médiatiques et dans les revendications de mouvements altermondialistes, comme l'agent d'une mondialisation économique et financière qui conduit à la perte des pouvoirs d'action économique antérieurement aux mains des acteurs du capitalisme national et des États. La culture est désormais au centre de ces mouvements. Armand Mattelart (2005: quatrième de couverture) souligne que « la mondialisation des industries culturelles a projeté les "produits de l'esprit" au cœur des négociations sur la libéralisation des échanges. Le thème de la diversité culturelle, longtemps retenu en lisière, a fait une entrée remarquée dans les grandes instances internationales ». Aujourd'hui comme hier, les législations nationales, y compris les dispositifs relevant de l'exception culturelle, n'entravent guère les flux culturels et informationnels internationaux, ni le libre investissement des capitaux étrangers (Bouquillion, 2004) dans les industries françaises de la culture et de la communication². Certes,

\footnotetext{
' Le caractère central du cinéma dans les politiques s'explique, d'une part, par la fragilité de cette économie de prototypes aux coûts de production élevés, d'autre part, par la capacité prêtée aux productions cinématographiques de contribuer à la construction des identités nationales, et aussi par la mobilisation des professionnels et les échos trouvés dans l'opinion.

2 Hormis quelques restrictions qui fixent un seuil à la présence des capitaux étrangers dans le capital des entreprises de presse et des entreprises exploitant des réseaux hertziens.
} 
des États comme la France veulent préserver leurs compétences dans le domaine culturel, pas seulement pour défendre l'identité nationale, mais aussi parce qu'il s'agit d'un des symboles de leur souveraineté. Mais, dans le même temps, ces États comprennent les limites d'une approche nationale et considèrent qu'une plus large libéralisation des industries de la culture et de la communication peut présenter des avantages. Les technologies de l'information et de la communication, tout particulièrement le Web, laissent croire que le cadre national est dépassé. La création et la diffusion de la culture et de l'information se construiraient désormais à l'échelle planétaire. Les questions culturelles sont traitées, non plus seulement par les États, mais aussi par des institutions internationales, comme l'UNESCO et même l'OMc, où la Commission européenne négocie pour le compte des États de l'Union. Par ailleurs, certains organismes dits de la société civile revendiquent une nouvelle gouvernementabilité, en particulier appliquée à la culture, qui dépasse le cadre étatique et même le système interétatique. Jean Tardif s'en fait l'écho.

D'autre part, l'économie des contenus culturels et informationnels connait de profondes mutations. Depuis le début des années 2000, la convergence ou, selon une expression plus appropriée, les articulations entre industries de la culture ${ }^{3}$ et industries de la communication ${ }^{4}$, tant annoncées depuis les années 80 , se développent. Or, ces mouvements, qu'on ne saurait réduire à la seule perspective empreinte de déterminisme technologique du développement du numérique, supposent de profonds réaménagements des filières dans chacune des deux industries, des transformations dans les pratiques et usages culturels, des mutations des réglementations et des dispositifs de régulation, et transforment les modes de transnationalisation de ces industries. Les acteurs industriels les mieux placés pour bénéficier de la redistribution des cartes en cours, au-delà de leurs intérêts divergents, appellent de leurs vœux ces transformations, notamment réglementaires. Dans nombre de pays, les pouvoirs publics comprennent qu'aujourd'hui les industries de la culture et de la communication, en cours d'articulation, sont un ensemble d'activités au poids économique important. Ils anticipent également une plus large articulation de ces industries avec le reste de l'économie. Avec le développement des places de marché électroniques, elles apparaissent comme étant au cœur de la diffusion, de la promotion et de la valorisation d'autres biens et services. De même, les thèmes des industries créatives et celui, voisin, de l'économie de l'immatériel (Lévy, Jouyet, 2006) tendent

\footnotetext{
3 Les industries de la culture regroupent les filières de la presse et de l'information, de l'édition, du cinéma et de l'audiovisuel et de la musique enregistrée.

4 II s'agit des filières des télécommunications et de l'offre d'accès à l'internet, de l'informatique et du Web et des matériels électroniques grand public.
} 
à accréditer l'idée d'un essaimage des logiques socio-économiques des industries de la culture et de la communication dans l'ensemble de l'économie. Les pouvoirs publics sont soucieux de défendre leurs intérêts économiques nationaux, notamment leurs champions industriels, face à de telles mutations. Ils sont pris en tenaille entre les demandes de protection en provenance de certains professionnels et les exigences d'une plus large libéralisation de la part de leurs champions industriels, la libéralisation étant perçue comme la condition sine qua non des transformations attendues de l'économie nationale, et ce bien au-delà, des domaines de la culture et de la communication. Les seconds risquent fort d'être plus entendus que les premiers.

Bref, la thématique de la diversité culturelle n'est-elle pas alors l'accompagnateur et un élément de légitimation de stratégies diverses, voire opposées? La complexité de la notion de diversité culturelle et son insertion dans des jeux de pouvoirs incitent à la considérer moins comme une « réalité » existant en dehors des acteurs et de leurs stratégies, que comme un ensemble de discours et de théories. En 1974, Michel de Certeau (in : Mattelart, 2005 : 3) remarque que « tout exposé concernant les problèmes culturels avance sur un sol de mots instables, il est impossible d'imposer une définition conceptuelle à ces termes: leurs significations tiennent à des fonctionnements dans des idéologies et des systèmes disparates ». Toute tentative de définition de la diversité culturelle et, en particulier, de production d'indicateurs semble dès lors caduque. On peut donc considérer les théories et les discours de la diversité, dans une perspective foucaldienne, tels des « discours de vérité » qui sont inséparables de systèmes de pouvoirs. Dans cette perspective, trois faits, en apparence non reliés, trouvent une cohérence.

Tout d'abord, le glissement sémantique de l'exception culturelle vers la diversité culturelle n'est pas anodin. La diversité culturelle ne présente pas sur le plan juridique les mêmes garanties que l'exception. Elle est largement reconnue, soit qu'on le regrette, soit qu'on s'en réjouisse, comme un gage de souplesse face à la relative rigidité et solidité juridique de l'exception culturelle. D'ailleurs, nombre d'industriels clament qu'ils conduisent une politique de la diversité. Jean-Marie Messier, encore dirigeant de Vivendi Universal, n'a pas été le seul à vanter les mérites de la diversité culturelle après avoir proclamé la mort de l'exception. Ensuite, nombre de travaux scientifiques, ou de rapports officiels, relatifs à la diversité concluent dans le sens d'une nécessaire révision des politiques publiques, voire à la réduction drastique de leur périmètre, et soulignent que c'est du côté des mécanismes du marché que l'avenir des industries nationales de la culture se joue. C'est d'ailleurs le cas des contributions de Joëlle Farchy et de Jean Tardif. D'autres, telle Françoise Benhamou (2006), soulignent que les politiques publiques sont contre-productives du point de vue de la diversité. 
Enfin, des auteurs, d'horizons idéologiques pourtant parfois opposés, ainsi que des rapports officiels et des discours d'acteurs économiques, traitent la question de la diversité en s'appuyant sur une définition anthropologique de la culture qui dépasse très largement les seules « productions culturelles ». Bien évidemment, la mondialisation transforme profondément les conditions de vie, de travail, les représentations du monde, d'où la référence à la culture au sens anthropologique.Tout un chacun peut souscrire au point de vue exprimé par Joëlle Farchy: «Parce que la diversité renvoie au respect d'identités et d'opinions diverses, elle est devenue un objectif politique bien au-delà de ce qu'appelleraient les caractéristiques économiques particulière de ces biens »). Cependant, les dispositifs juridiques s'appliquant aux productions culturelles se trouvent alors fragilisés. Mettre en perspective des productions culturelles et informationnelles - qui nécessitent des protections réglementaires du fait de leurs spécificités socio-économiques - et d'autres produits relevant des industries créatives - qui n'appellent pas de dispositifs de soutien -, conduit à rendre moins crucial le maintien des réglementations. A fortiori lorsque les produits culturels et informationnels sont, au nom de leur commune participation à la construction des identités, mis sur le même plan que les croyances ou les comportements et autres «traits » culturels relevant de la culture au sens anthropologique du terme, les dispositifs de l'exception culturelle perdent tout sens.

En somme, cohabitent au sein des discours sur la diversité culturelle, à la fois des considérations très générales relatives à la culture dans la mondialisation et des réflexions sur les enjeux économiques des réglementations relatives aux industries de la culture. Une telle cohabitation concourt à légitimer la plus large libéralisation demandée par les industriels. Comment ces mutations industrielles, aidées par les discours de vérité que sont les théories et discours de la diversité, pèsent-elles sur l'économie des contenus? Telle est l'interrogation centrale qui nous permettra de discuter certains des points de vue défendus ou présentés par Joëlle Farchy et Jean Tardif et, le cas échéant, d'apporter des contrepoints et des compléments. Cette interrogation sera conduite au long de trois volets successifs, considérés généralement comme étant les questions essentielles à la compréhension des mutations des contenus : le fonctionnement oligopolistique des marchés de la culture et de la communication, l'entrée des acteurs des industries de la communication dans les contenus, les politiques publiques et de régulation. Nous nous appuyons sur les résultats d'une récente recherche collective ${ }^{5}$.

5 II s'agit d'une recherche sur la diversité culturelle, coordonnée par la Maison des sciences de l'homme Paris Nord, et financée dans le cadre de l'appel à projets du ministère de la Culture: «La diversité dans les filières d'industries culturelles ». De plus amples informations sur cette recherche sont disponibles sur le site de l'Observatoire international des mutations des industries culturelles (http://www.observatoire-omic.org/). 


\section{Oligopoles et économie des contenus}

Les marchés de la culture et de la communication présentent des modes de fonctionnement spécifiques et complexes. Ils sont organisés sous la forme d'oligopoles à franges. Autrement dit, dans chacune des filières des contenus, en particulier dans les filières du cinéma et de l'audiovisuel, un petit nombre d'acteurs, les plus importants, souvent nommés majors, concentrent l'essentiel de la production de contenus dits premium, c'est-à-dire les plus coûteux et censément les plus susceptibles de générer des profits. Ces acteurs disposent également d'une mainmise sur la distribution-diffusion à l'échelle nationale et, plus encore, à l'échelle internationale. Comme dans un goulot d'étranglement, les contenus que les diffuseurs-distributeurs jugent potentiellement les moins rentables, n'accèdent pas au stade de la diffusion, en particulier la grande masse des contenus offerts par les petits acteurs. Ceux-ci ont pour rôles principaux la prise de risques artistiques, notamment la découverte et la « construction » de nouveaux talents. Ils contribuent de manière déterminante au nécessaire renouvellement permanent de l'offre. Néanmoins, avec ce système, nombre de contenus produits ne sont pas diffusés. De surcroît, une masse importante de produits ne sont pas consommés, car les consommations se concentrent sur un nombre extrêmement faible de produits. Si ces éléments sont globalement partagés, les points de vue cités par Joëlle Farchy appellent, selon nous, trois nuances qui conduisent à mettre l'accent sur le lien entre l'organisation des filières et la gestion du risque, sur la nécessité de prendre en compte les logiques socio-économiques des industries culturelles et la financiarisation, et sur la dimension idéologique des théories relatives aux relations entre concentration et diversité.

D'abord, la déperdition successive entre les contenus produits et les contenus consommés peut être considérée comme le signe d'un dysfonctionnement de l'économie des contenus et la preuve que l'organisation des filières défavorise la diversité. Les théories des industries culturelles ${ }^{6}$ estiment qu'il s'agit là plutôt d'un mode de gestion du risque. Le caractère aléatoire de la valorisation des biens culturels est l'une des caractéristiques marquantes de ces activités. II s'explique par le fait que les productions culturelles sont des biens symboliques dont la valeur est liée au travail artistique et intellectuel. II s'explique aussi par le caractère relativement peu rationalisé des procès de production (Mœglin, 2007). Comme le souligne Joëlle Farchy, chaque filière dispose d'une organisation permettant de faire face à ce risque. Le rôle des

\footnotetext{
6 II s'agit d'un ensemble de propositions théoriques relatives aux industries culturelles construites, à partir des années 70, par un ensemble de chercheurs européens, Nord et Sud-américains, principalement issus des sciences de l'information et de la communication ou des Media Studies, et qui tous, se réclament d'une approche critique renouvelant les théories de l'école de Francfort.
} 
petits acteurs a déjà été souligné. La « dialectique du tube et du catalogue » (Miège, 1984) est une modalité également importante dans les filières proches du modèle éditorial, notamment le disque et le livre.

Ensuite, on peut s'accorder sur le fait que les liens entre la concentration et la diversité sont complexes. La concentration ne rime pas nécessairement avec standardisation. Toutefois, des arguments différents peuvent être avancés de part et d'autre. De nombreuses références sont faites aujourd'hui par des économistes aux travaux de Peter Steiner (1952) pour justifier le degré élevé de concentration dans les différentes filières des industries de la culture et de la communication, alors que cet auteur a travaillé sur la radio américaine dans les années 50. II est souligné que la concentration est un facteur de diversité puisqu'elle permet de combiner, au sein du même groupe industriel, des stratégies grand public et des offres de niches. Des péréquations entre produits très rentables et d'autres, à faible rentabilité, s'opéreraient au sein des groupes industriels, alors que dans des marchés plus concurrentiels les acteurs viseraient tous les segments grand public qui sont les plus rémunérateurs.

Outre que les travaux de Peter Steiner n'expliquent pas pourquoi des groupes renonceraient à maximiser leur rentabilité au profit d'une logique de diversité culturelle, les théories des industries culturelles ont une approche différente. Les « modèles socio-économiques » ${ }^{7}$, élaborés par des chercheurs en communication, rendent compte des modalités spécifiques (par rapport aux autres activités économiques) et différenciées (entre elles) qui régissent le fonctionnement des industries de la culture. La concentration ne fait pas table rase des modalités spécifiques d'organisation des filières - bien qu'elle puisse concourir aux transformations de celles-ci sur le temps long - pour soumettre, avec la financiarisation, les productions culturelles à la loi d'une supposée standardisation. Ainsi, à la suite de Bernard Miège (2000: 18), peut-on remarquer qu'il est impossible d'induire des liens mécaniques entre concentration des marchés et taille des acteurs industriels d'un côté, et les formes de la marchandisation et de l'industrialisation des productions de l'autre : «De la prise de contrôle financier à la production de la culture et à celle de l'information, interviennent toute une série de médiations qu'on ne saurait a priori passer sous silence $\gg$. La financiarisation ${ }^{8}$, quant à elle, a fait

\footnotetext{
7 Pour une présentation synthétique des modèles, voir P. Mœglin (2007).

8 La financiarisation désigne le passage des économies nationales d'une logique de « cœurs financiers » à une économie de «marchés financiers » (Morin, 1999), dominée par des acteurs anglo-saxons. Les cœurs financiers sont formés par de grandes banques ou assurances qui se regroupent en deux ou trois ensembles et qui, à elles seules, détiennent une large part du capital des entreprises cotées en bourse dans un espace national donné. Par extension, elle désigne aussi la montée de la participation des acteurs financiers internationaux, notamment anglo-saxons, au sein du capital des groupes industriels et l'accroissement de leur influence sur la gestion des groupes.
} 
l'objet de plusieurs travaux qui relativisent sa portée, au nom de la prégnance des modes d'organisation des filières dont rendrent compte les modèles, mais aussi parce que la gouvernance financière, dans les industries de la culture, du moins en France, ne connaît que des développements limités (Robin, 2003), même si ce thème est très présent dans la communication financière des entreprises (Bouquillion, 2005). Certains enjeux pour l'économie des contenus de la concentration ou de la financiarisation peuvent néanmoins être relevés. Les intégrations capitalistiques, mais aussi les alliances, peuvent dessiner un « espace sécurisé » au sein duquel les transactions s'effectuent aisément entre des contenus étrangers, en particulier américains, et des structures de diffusion, notamment en Europe. Tel était le projet industriel envisagé par Jean-Marie Messier pour Vivendi Universal qui, d'ailleurs, diminuait grandement l'intérêt stratégique des importants investissements de Canal + dans le cinéma français. Concentration et financiarisation contribuent ainsi à maintenir une organisation transnationale des rapports entre groupes industriels qui fait des uns, par exemple des acteurs tels TFI, des relais assurant la diffusion des produits des autres (les acteurs américains des contenus). D'autre part, la volonté de maintenir des cours de bourse élevés oblige à verser des dividendes importants et réguliers aux actionnaires. \| est alors logique de rechercher un raccourcissement des délais de retour sur investissement. La remontée des recettes vers l'amont de la filière, en particulier la production des contenus, peut en être affectée.

Enfin, certains des travaux cités par Joëlle Farchy relatifs aux flux culturels internationaux nous semblent pouvoir être qualifiés de discours de vérité. C'est en particulier le cas de l'approche de Tyler Cowen (2002: I5), selon laquelle « le commerce culturel augmente le menu de choix disponible pour chacune des sociétés engagées dans l'échange, mais diminue la différence entre menus de choix de ces deux sociétés ». Cette représentation fait de l'échange la clé de la diversité culturelle. Elle conduit à disqualifier les volontés de certains pays, dont la France, de défendre leurs productions nationales. Ces politiques qui sont qualifiées de nationalisme culturel apparaissent comme le signe d'une fermeture au monde. Elles sont accusées de réduire la diversité. Ces travaux ont rencontré des échos favorables chez certains économistes français. « On peut d'autant mieux s'accommoder de la réduction de la diversité entre les sociétés que les produits plus marchands, plus homogénéisés, seraient plus facilement exportables », souligne Françoise Benhamou (2006 : 256). La définition de la culture sur laquelle s'appuie Tyler Cowen est très large. Elle s'étend à un ensemble de biens et de services présentés comme disposant d'une valeur symbolique à l'instar des produits culturels. Les produits alimentaires, par exemple, peuvent en faire partie. Ainsi, noyées dans un vaste ensemble, les productions culturelles ne sont-elles plus une catégorie pertinente justifiant des politiques publiques spécifiques. De même, les Cultural Studies et les approches d'Arjun Appaduraï, en mettant 
l'accent non pas sur les conditions de production des produits culturels mais bien sur la réception et son « autonomie » par rapport à l'offre, font apparaître les dispositifs réglementaires qui pèsent sur l'offre comme des enjeux d'ordre secondaire. Autrement dit, si les publics peuvent se réapproprier les œuvres, selon des clés qui leur sont propres, alors la domination des produits culturels américains sur les marchés mondiaux des programmes n'est qu'un danger très relatif pour les identités culturelles. Ainsi Tristan Mattelart (2007), dans une perspective généalogique, montre-t-il combien les fondements de l'économie politique critique de la communication, laquelle dénonce depuis les années 70 les flux culturels transnationaux inégaux, sont remis en cause par ces approches. Celles-ci peuvent faire l'objet d'instrumentalisation à des fins de contestation des politiques nationales de la culture et de la communication.

\section{Les acteurs des industries de la communication et l'économie des contenus}

Avec les mutations du Web, en particulier l'augmentation des taux d'équipement des ménages en ordinateurs connectés à l'internet à haut débit, les stratégies industrielles tendant à articuler les industries de la culture et les industries de la communication trouvent désormais un cadre favorable. Ces mouvements génèrent une situation de grande incertitude ; aucun acteur industriel, aucune instance de régulation n'est en mesure de contrôler ou de prévoir les développements futurs. Cependant, trois enjeux pour l'économie des contenus peuvent être d'ores et déjà envisagés. Si les discours dans l'air du temps envisagent ces mouvements comme une opportunité pour les contenus, pour notre part, nous considérons que ces mutations n'apportent guère de ressources supplémentaires destinées à la production de contenus. Ces ressources risquent même de réduire et de se concentrer sur les contenus dits premium, les hits, tandis que les phénomènes de rationalisation s'intensifient.

Premièrement, l'entrée des acteurs de la communication dans l'économie des contenus conduit à la multiplication de supports en émergence et au développement de stratégies multi supports (qui conduisent à diffuser un même contenu sur plusieurs supports). Certes, les stratégies multi supports ne sont pas nouvelles. L'histoire du cinéma en témoigne, mais aujourd'hui elles s'intensifient et se complexifient. Le déploiement des supports émergents favorise-t-il la production de contenus originaux? Et, dans l'affirmative, de quels types de contenus s'agit-il ? II est difficile de répondre à cette question compte tenu du caractère très évolutif des stratégies des acteurs. Plusieurs éléments peuvent néanmoins être avancés. 
Les ressources collectées par les supports émergents sont encore limitées. D'une part, toutes les filières ne sont pas également concernées par l'essor des supports émergents. À cet égard, la situation des filières de la presse, de l'information et de la musique enregistrée s'oppose à celle de la filière du livre. D'autre part, même dans les filières les plus concernées, le déploiement des supports émergents ne se traduit pas nécessairement par la réalisation de chiffres d'affaires importants. Par exemple, les « plates-formes » musicales légales ne réalisent qu'une part encore faible du chiffre d'affaires total de la filière. Dans le même temps, les échanges illégaux de pairs à pairs contribuent à la baisse des recettes générées par les ventes de disques, du fait des effets de substitution qui s'opèrent entre les achats de disques et les téléchargements illégaux mais aussi parce qu'ils ont incité à baisser le prix moyen des disques. Notons également que la domination qu'exercent les acteurs de la communication sur ces supports concourt à fixer les prix de référence à un niveau jugé faible par les acteurs des contenus. L'exemple du fichier musical à 0,99 dollars vendu sur ITunes est bien connu. Dans le domaine de la presse et de l'information, les transferts des budgets des annonceurs des titres papiers vers les sites du Web sont déjà bien réels. Une des questions qui se pose est celle de la part respective des ressources collectées par les sites Web des titres « papier » et par les pure players du Web. En effet, la notion de contenus prend un sens très différent dans un cas et dans l'autre. À titre d'exemple, les sites offerts par les titres de la presse magazine de Lagardère et le site AuFéminin. com ont des approches très différentes de l'information journalistique (Rebillard et al., 2007). La situation du cinéma et de l'audiovisuel est particulière puisque les supports en place, dans un pays comme la France du moins, conservent un rôle tout à fait central. La plupart des acteurs dominants de l'audiovisuel et du cinéma développent des stratégies en direction des supports émergents. Néanmoins, en fonction de leur origine - industries de la culture ou de la communication -, et de leur place dans la filière, amont ou aval, ils ont des anticipations assez différentes du devenir des supports émergents, de l'ampleur et du rythme de leur développement. Dans cette filière comme dans les autres, les acteurs de la communication semblent jouer un rôle moteur dans le déploiement des supports émergents par rapport aux acteurs des industries de la culture.

Ensuite, les acteurs industriels des filières cinématographiques et audiovisuelles semblent vouloir limiter la production de contenus spécifiques pour des supports, tels les outils mobiles, très différents des principaux supports en place, dès lors que ces contenus ont un coût de production significatif (Bouquillion et al., 2007). Les professionnels que nous avons interrogés préfèrent recourir à des contenus de niche ou diffuser des contenus conçus pour d'autres supports. 
Enfin, l'offre de contenus originaux ne présente pas le même intérêt stratégique pour la valorisation des différents supports. Les opérateurs des supports émergents ne financent la production de contenus originaux que lorsqu'ils ne peuvent pas en obtenir autrement et que ces contenus sont nécessaires à la différenciation de leurs offres. C'est en particulier le cas de fournisseurs d'accès à Internet, tel Orange, qui veulent distinguer leur offre triple play de celle de leurs concurrents. À cette fin, Orange cherche à offrir des films originaux offerts en exclusivité en Video On Demand ${ }^{9}$ (VOD). Or, compte tenu de la stratégie d'exclusivité conduite par Canal + , Orange développe sa filiale de production cinématographique pour disposer de ses propres films mais aussi pour faire pression sur Canal +. Sur le plan européen, la tendance est en revanche à la nonexclusivité, avec des perspectives de réduction du nombre de plateformes différentes (Observatoire européen des médias, 2007 : 24). D'autres supports peuvent facilement bénéficier d'une articulation avec des contenus sans prendre en charge leur production. C'est en particulier le cas des matériels, notamment des baladeurs. Les acteurs des matériels ont largement profité des échanges de pairs à pairs illégaux et n'ont consacré que des ressources marginales à la production de contenus, y compris ceux qui offrent des bases d'accès à des contenus, tel Apple avec ITunes.

Deuxièmement, les supports émergents conduisent-ils à un allongement de la durée de vie des produits? De même, les consommations sontelles plus dispersées ou toujours concentrées sur un petit nombre de productions phares? Deux éléments de réponse sont généralement présentés. L'un ayant trait à la chronologie des médias qui concerne pour l'essentiel les produits cinématographiques et audiovisuels, et qui tend à se réduire. Les dispositifs réglementaires ou interprofessionnels organisant la chronologie des médias sont remis en cause aujourd'hui, tant par les échanges de pairs à pairs, que par les stratégies de certains acteurs. Dès que les films et les séries sont diffusés une première fois, voire avant, les fichiers électroniques de ces programmes sont disponibles sur des sites d'échanges. Ils perdent ainsi une large part de leur « originalité » lorsqu'ils sont diffusés sur des chaînes payantes ou hertziennes, ce qui rend celles-ci moins attractives pour les abonnés et les annonceurs. De même, une chronologie très structurée des médias, et qui prévoit de longs délais entre les diverses «fenêtres », n'avantage que les acteurs présents dans les différents stades de valorisation. Les autres acteurs ont intérêt au raccourcissement de ces délais. Ils espèrent ainsi mieux profiter de la promotion qui, généralement, entoure le film ou la série lors de son « lancement ». Ces deux raisons plaident pour la disparition, à terme,

9 Vidéo à la demande. 
de la chronologie des médias ou, du moins, pour son assouplissement, ce qui évidemment rend plus difficile la valorisation des contenus. Ce mouvement se dessine à l'échelle européenne avec le projet de Directive « Services de médias audiovisuels » qui maintient le principe d'une chronologie des médias en l'étendant aux services dits non-linéaires mais en l'assouplissant (Observatoire européen des médias, 2007 : 72-73).

D'autre part, les diffusions électroniques conduisent, selon les premières études, à la fois à disperser les ventes en un plus grand nombre de titres et à allonger la durée de vie de certains produits, comme l'affirme la théorie de la longue traîne développée par Chris Anderson (source: http://thelongtail.com). Toutefois, les professionnels soulignent clairement que les recettes dégagées sur les titres à faibles ventes ne suffisent pas à financer la production de contenus originaux. La Fondation internet nouvelle génération (FING, 2006-2007:40) l'a constaté en matière musicale. De même, la longue traîne s'applique plus à des œuvres déjà produites qu'un grossiste chercherait à vendre. Sa portée pour l'économie de la création en est d'autant plus réduite : «Pour le dire autrement, la «longue traîne» est une théorie avant tout destinée et adaptée à des distributeurs qui gèrent un stock et de nombreuses références. Mais cette théorie ne dit rien du destin d'un artiste individuel, ni même d'un label qui promeut un petit nombre d'artistes » (ibid.).

Troisièmement, quels sont les enjeux pour la diversité culturelle des stratégies de rationalisation des contenus ? Les stratégies de rationalisation des procès de travail et des organisations ne sont pas nouvelles au sein des industries de la culture, mais elles tendent à s'intensifier aujourd'hui à l'occasion des mouvements d'articulation entre industries de la culture et industries de la communication. Franck Rebillard (Rebillard et al., 2007) évoque ainsi la montée d'une logique «gestionnaire » au sein de la presse et de l'information liée au développement des publications sur le Web. Ces phénomènes s'observent également dans les filières des contenus les plus concernées par les articulations avec les industries de la communication, en particulier la musique enregistrée, le cinéma et l'audiovisuel. Quatre stratégies s'articulent: le développement du marketing, dit amont, le recours à des indicateurs financiers, l'externalisation des tâches de création et les nouveaux développements du marketing, dit aval. Néanmoins, les tentatives de rationalisation des contenus de la part des directions des groupes industriels sont d'autant plus difficiles à mettre en œuvre que la valeur d'un produit culturel est liée à l'insertion de travail artistique ou intellectuel. De fait, on observe, en particulier dans la presse et l'information (Rebillard et al., 2007), qu'il s'agit d'abord de rationaliser les coûts de production, d'inciter les équipes de création et de production à faire les choix les plus « économes 》, et à leur faire intérioriser la contrainte de la maîtrise des coûts de production. Une 
telle maitrise est d'autant plus impérative que les industries de la culture connaissent des mouvements tendanciels de hausse des coûts, hausse à laquelle les coûts de promotion concourent activement.

\section{Politiques publiques et économie des contenus}

La question des politiques, au cœur des contributions de Joëlle Farchy et de Jean Tardif, appelle trois commentaires. Les auteurs soulignent que les dimensions financière et idéologique des politiques publiques culturelles sont oubliées dans nombre de travaux de recherche, tandis qu'à l'heure où les politiques de soutien sont contestées tant par les auteurs que par les acteurs économiques, leur bilan n'a pas été dressé de manière suffisamment complète.

On ne peut que constater que la convention de 2005 n'offre que des garanties limitées, les États-Unis recourant aux accords bilatéraux comme l'a montré Joëlle Farchy. Plus largement, la culture est au cœur de rapports de force. Les discours dénonçant le patriotisme culturel français oublient, comme le remarque Jean Tardif, que depuis longtemps les États-Unis soutiennent leurs productions culturelles nationales. De même, des phénomènes de domination dans des zones régionales peuvent s'exercer. L'exemple de la Corée a été cité. Mais un aspect pourtant important de l'économie-monde de la communication est fréquemment oublié, la dimension financière. D'une part, à l'occasion des rapprochements entre groupes industriels, la dépendance de ceuXci vis-à-vis de la sphère financière, dominée par des institutions anglosaxonnes, s'accroît (Bouquillion, 2008). Ils ont besoin de prêts, de réaliser des émissions d'obligations, d'augmenter leur capital, voire d'obtenir de bonnes notations. Les pouvoirs des acteurs financiers sur le management des acteurs industriels s'en trouvent renforcés. Les institutions financières contribuent à définir les contours des nouveaux ensembles. Elles orientent les grands choix stratégiques et la nomination des responsables. D'autre part, elles deviennent les véritables «propriétaires » des pôles ou des groupes. Elles sont ainsi en mesure de faire remonter vers elles les profits. Cette domination conduit également à rattacher financièrement les industries de la culture et de la communication à la sphère supérieure des acteurs du capitalisme, tout en conservant les distinctions entre espaces nationaux et aires géo-culturelles. La financiarisation permet aussi de maintenir, sans dommage pour le capitalisme, des conflits entre

${ }^{14}$ En majuscules dans le texte original. Courriel ( $14 / 09 / 0$ I). 
acteurs industriels puisque ceux-ci sont tous, peu ou prou, rattachés aux acteurs financiers qui sont leurs propriétaires, leurs conseillers ou leurs créditeurs. L'approfondissement de la dimension financière est au cœur du renforcement de la hiérarchie existant au sein de l'économiemonde de la communication. En s'inspirant de Fernand Braudel (1985) et d'Immanuel Wallerstein (1985), on peut considérer qu'elle est structurée et hiérarchisée en différents cercles concentriques. Or, les politiques publiques nationales ou les instances supranationales ne s'intéressent guère à ces questions.

La question des droits de propriété intellectuelle est fréquemment évoquée. Joëlle Farchy montre comment ces droits peuvent être, soit considérés comme les moteurs du développement des productions, soit comme un frein. Les intérêts industriels sont plutôt divergents. Afin de tenter de lutter contre les échanges illégaux de fichiers, les acteurs des contenus, tout particulièrement les industriels du disque et leurs syndicats professionnels, concourent fortement, depuis le début des années 2000, à la construction de l'image de l'usager-délinquant (Vandiedonck, 2007) et de sa victime, l'artiste qui perd ses moyens de créer. De même, des industriels, des contenus comme des industries de la communication, envisagent, grâce à la dématérialisation, de valoriser certains produits en proposant un droit d'usage éphémère et non plus inscrit sur un support durable. La construction de la figure du « pirate » peut alors aider à légitimer la transformation des pratiques culturelles ancrées depuis fort longtemps - l'achat d'un livre ou d'un disque permet un accès permanent au contenu - et à faire évoluer les logiques socioéconomiques qui régissent ces productions (Mœglin, 2003). Depuis la seconde moitié des années 2000, avec la montée en puissance des grands sites du Web collaboratif et le développement de jeux, tel Second Life, se développe un discours différent, celui de l'usager-créateur. Des acteurs, généralement issus des industries de la communication, dont les modes de valorisation ne reposent pas principalement sur la vente de contenus aux consommateurs finaux, se présentent comme offrant un «cadre » pour que les usagers puissent donner libre cours à leur créativité en développant des « users-created contents ${ }^{10} \gg$. Ces discours rencontrent les intérêts d'autres industriels de la communication qui vendent des matériels ou des abonnements à des connexions triple play, ou dont le financement repose sur la publicité, le courtage ou la vente d'informations marketing. L'écart entre les deux groupes d'acteurs peut se réduire à l'avenir si les acteurs de la communication estiment qu'ils ont intérêt à offrir des contenus originaux pour distinguer leur offre.

${ }^{10}$ Contenus créés par les utilisateurs. 
Alors que les politiques nationales sont attaquées, force est de constater que leur bilan n'a pas été dressé de manière exhaustive filière par filière. Certes, elles n'empêchent pas les flux culturels internationaux. Certaines de leurs inflexions récentes peuvent d'ailleurs concourir à plus articuler acteurs économiques nationaux et étrangers comme l'illustre la transformation des règles de nationalité pour bénéficier des aides françaises en matière audiovisuelle. La venue de producteurs étrangers aide à amortir les coûts des systèmes de production existant en France. Mais sans elles, les niveaux d'activité des acteurs nationaux seraient moindres, et les équilibres entre l'amont, la production des contenus et l'aval, leur diffusion, seraient certainement très différents. C'est d'ailleurs ce dernier point qui explique pourquoi les stratégies de certains acteurs économiques de la culture et de la communication tendent à remettre en question ces politiques. Les filières cinématographiques et audiovisuelles en offrent une illustration. Aujourd'hui, les dispositifs de soutien posent deux problèmes principaux.

L'un relatif au fait que l'entrée des acteurs des industries de la communication dans l'économie des contenus n'est pas prévue dans le cadre en place. Actuellement, seuls les acteurs de l'audiovisuel sont soumis à ces obligations, notamment aux règles d'investissement dans la production indépendante et aux quotas de diffusion. Or, l'extension de celles-ci aux supports émergents et aux nouveaux entrants ne va pas de soi. La révision de la directive «Télévision sans frontière », en cours, prévoit d'assujettir les offres dites non linéaires de contenus audiovisuels (y compris cinématographiques) à un système d'obligations allégé par rapport à celui qui pèse sur les services dit linéaires. Néanmoins, il est parfois difficile de qualifier les services offerts sur les supports émergents et de définir de manière précise la contribution des divers acteurs. Des fournisseurs d'accès à l'internet, des opérateurs de télécommunications ou des acteurs du Web, qui ne sont pas actuellement soumis à ces obligations, ne souhaitent pas l'être. Seuls ceux qui en ont les moyens financiers voient là une façon de distinguer leurs offres de celles de leurs concurrents. Or, il est impossible pour les autorités françaises de procéder de manière autoritaire vis-à-vis des nouveaux entrants qui pourraient se tourner vers les autorités européennes. Pourtant, il est impossible de ne pas assujettir les « anciens » et les « nouveaux » acteurs de l'audiovisuel aux mêmes règles car cela créerait une distorsion de concurrence contraire au droit en vigueur. Le maintien, la suppression, ou l'allègement des dispositifs de soutien sont donc en jeu.

Ensuite, seul l'accès aux programmes premium (dont les contenus étrangers achetés) intéresse les chaînes de télévision, tant Canal + que les chaînes commerciales à financement publicitaire. Les principaux acteurs des filières cinématographiques et audiovisuelles, ainsi que les 
nouveaux entrants, dont les intérêts sont situés en aval des filières, peuvent avoir intérêt à remettre en cause une réglementation qui vise avant tout à assurer la remontée des ressources vers l'amont et, ainsi, à assurer le financement de la production. Les obligations d'investissement et de diffusion représentent de lourdes charges financières, tandis qu'elles peuvent freiner les évolutions des grilles de programmes des chaînes commerciales vers des programmations encore plus commerciales. Les acteurs semblent d'ailleurs plus demander un aménagement du système que sa totale remise en question, tandis que les producteurs indépendants comprennent qu'il sera difficile de maintenir la réglementation existante (Bouquillion et al., 2007).

\section{Conclusion}

Au terme ce trop rapide examen des mutations en cours des industries de la culture et de la communication, quatre tendances de long terme, inscrites dans l'histoire de ces industries, semblent se dégager. Le mouvement ancien qui pousse le centre de gravité des filières vers l'aval, semble se poursuivre à l'occasion des articulations croissantes entre industries de la culture et de la communication. Lors de la clôture du colloque «Mutation des industries de la culture, de l'information et de la communication » tenu en septembre 2006 à la Plaine Saint-Denis"', Nicholas Garnham soulignait que les filières des industries de la culture, d'un côté, et des industries de la communication de l'autre, demeurent très distinctes. II est vrai que la taille des marchés, les niveaux des chiffres d'affaires ou de la valorisation boursière des grands acteurs industriels sont sans commune mesure, de part et d'autre. Ainsi les stratégies déployées dans les contenus par les acteurs de la communication visentelles principalement à asseoir leur position dans leur domaine d'activité principal. Réaliser des gains liés à la valorisation directe des contenus, sans être une question négligeable, ne semble pas être leur objectif premier, du moins à ce jour. L'inclusion des contenus dans des cycles de production qui les dépassent, initiée par la publicité dès le XIXe siècle, s'accroît donc singulièrement.

\footnotetext{
' Le colloque international «Mutation des industries de la culture, de l'information et de la communication » a été organisé par la msh Paris-Nord, la msh des Alpes, la msh d'Aquitaine, la msh de Paris, la msh Nord-Pas de Calais et le Gricis (Groupe de recherche interdisciplinaire sur la communication, l'information et la société) de l'université du Québec à Montréal. II s'est inscrit dans le cadre de l'Action concertée incitative (aci) du réseau des msh. Pour toute information complémentaire, voir http://www.observatoireomic.org/colloque-icic/index.php
} 
L'organisation dans une logique d'économie-monde des industries de la culture et de la communication se maintient. La financiarisation y concourt désormais activement. La concentration et la financiarisation, à défaut de peser directement sur les contenus, contribuent à offrir un cadre favorable aux flux culturels internationaux inégaux et à orienter les ressources collectées non pas en direction des contenus mais soit vers les investissements en aval des filières soit en rémunérations pour les acteurs de la sphère financière.

La polarisation tendancielle des contenus entre hits et contenus de niche semble s'accentuer. Certes, jamais autant de contenus n'ont été produits, mais il s'agit pour l'essentiel de contenus de niche produits à moindres frais par les petits acteurs économiques ou directement par les consommateurs. De leur côté, les acteurs dominants restreignent leurs investissements dans la production des contenus destinés à devenir des hits.

Les politiques publiques nationales de la culture et de la communication ne sont pas seulement attaquées par certaines des théories de la diversité, œuvrant alors dans une logique de discours de vérité, mais leur remise en cause est inscrite dans les stratégies mêmes des acteurs économiques, y compris de nombre des acteurs dominants des contenus.

\section{Références}

Benhamou F., 2006, Les dérèglements de l'exception culturelle, Paris, Éd. Le Seuil.

Bouquillion Ph., 2004, « La propriété étrangère dans les industries de la culture et de la communication en France », Les Cahiers Médias, I5, pp. 63-92.

- 2005, «La constitution des pôles des industries de la culture de la communication. Entre coups financiers et intégration de filières industrielles », Réseaux, | 3 |, pp. | | |- | 44.

- 2008, Les industries de la culture et de la communication. Les stratégies du capitalisme, Grenoble, Presses universitaires de Grenoble.

Brigaud-Robert N., Combès Y., Rodionoff A., 2007, « La diversité dans les filières d'industries culturelles: les filières cinématographiques et audiovisuelles », in : Bouquillion Ph., dir., Rapport au Département des études, de la prospective et des statistiques, ministère de la Culture et de la Communication.

Braudel F., 1985, La dynamique du capitalisme, Paris, Flammarion.

Cabedoche B., Damian B., Smyrnaïos N., 2007, «La diversité dans les filières d'industries culturelles : les filières de la presse et de l'information », in : Rebillard Fr., dir., Rapport au département des études, de la prospective et des statistiques, ministère de la Culture et de la Communication.

Certeau M. de, 1974, La culture au pluriel, Paris, Chr. Bourgeois.

Cowen T., 2002, Creative destruction, how globalization is changing the world's cultures, Princeton, Princeton University Press. 
Fondation internet nouvelle génération, 2006-2007, « Débat public, Musique \& Numérique : créer de la valeur par l'innovation ».

Lévy M., Jouyet J.-P., 2006, L'économie de l'immatériel, Rapport au ministre de l'Économie et des Finances.

Mattelart A, 2005, Diversité culturelle et mondialisation, Paris, Éd. La Découverte.

Mattelart Tr., 2007, Déconstruire la diversité culturelle, Rapport au Département des études, de la prospective et des statistiques, ministère de la Culture et de la Communication.

Miège B., 1984, « Postface à la seconde édition », pp. 199-214, in : Huet A., lon J., Lefebvre A., Miège B., Péron R., dirs, Capitalisme et industries culturelles, Grenoble, Presses universitaires de Grenoble.

- 2000, «À l'arrière-plan des récents mouvements de concentration », Les dossiers de l'audiovisuel, 94, pp. 18-20.

Mœglin P., 2007a, « Repenser l'usager, pour un universalisme concret », pp. 17-31, in : Lacroix J.-G., Gaëtan Tremblay G., dirs, 200 I Bogues. Globalisme et pluralisme. Tome 2 : Usages des TIC, Québec, Presses de l'université Laval.

- 2007b, « Des modèles socio-économiques en mutation 》, pp. I5 |-162, in : Bouquillion Ph., Combès Y., dirs, Les industries de la culture et de la communication en mutation, Paris, Éd. L'Harmattan.

Morin F., (entretien avec), 1999, « Où va le capitalisme? », La lettre du LEREPS, 7.

Observatoire européen des médias, Direction du développement des médias, 2007, « La vidéo à la demande en Europe », mai, 32 p.

Regourd S., 2002, L'exception culturelle, Paris, Presses universitaires de France.

Robin Ch., 2003, « La gestion et le contenu des livres. Les enjeux de l'information et la communication », Les Enjeux, http://www.u-grenoble3.fr/lesenjeux/

Steiner P. O., 1952, « Program Patterns and Preferences and the Workability of Competition in Radio Broadcasting », Quaterly Journal of Economics, 66(2), pp. 194-223.

Vandiedonck D., «L'industrie de la musique recomposée », pp. 91-98, in: Bouquillion Ph., Combès Y., dirs, Les industries de la culture et de la communication en mutation, Paris, Éd. L'Harmattan.

Wallerstein I., 1985, Le capitalisme historique, trad. de I'anglais par Ph. Steiner et Chr.Tutin, Paris, Éd. La Découverte/Syros, 2002. 